\title{
Spin fluctuation and small polaron conduction dominated electrical resistivity in $\mathrm{La}_{0.875} \mathrm{Sr}_{0.125} \mathrm{MnO}_{3}$ manganite nanostructures
}

\author{
K K CHOUDHARY*, N GUPTA, N KAURAV ${ }^{1}$, S KATIYAL ${ }^{2}$ and S K GHOSH ${ }^{3}$ \\ Department of Physics, National Defence Academy, Khadakwasla, Pune 411 023, India \\ ${ }^{1}$ Department of Physics, Holkar Science College, A-B Road, Indore 452 001, India \\ ${ }^{2}$ School of Electronics, Devi Ahilya University, Khandwa Road Campus, Indore 452 001, India \\ ${ }^{3}$ School of Studies in Physics, Vikram University, Ujjain 456 010, India
}

MS received 13 April 2013; revised 28 June 2013

\begin{abstract}
The anomalous temperature-dependent electrical resistivity $\rho(T)$ of $\mathrm{La}_{0.875} \mathrm{Sr}_{0.125} \mathrm{MnO}_{3}$ manganite nanoparticles (particle size $18 \mathrm{~nm}$ ) is theoretically analysed. $\rho(T)$ exhibits semiconducting phase in lowtemperature regime $(20 \mathrm{~K}<T<53 \mathrm{~K})$, shows a minima near $53 \mathrm{~K}$ and increases with $\mathrm{T}$ at high temperatures ( $53 \mathrm{~K}<T<170 \mathrm{~K})$. The resistivity in metallic phase $(T>53 \mathrm{~K})$ is theoretically analysed by considering the strong spin-fluctuation effect, which is modelled using Drude-Lorentz type function. In addition to the spin fluctuation-induced contribution, the electron-phonon and electron-electron $\rho_{\mathrm{e}-\mathrm{e}}(T)=B T^{2}$ contributions are also incorporated for complete understanding of experimental data. The contributions to the resistivity by inherent acoustic phonons $\left(\rho_{\mathrm{ac}}\right)$ as well as high-frequency optical phonons $\left(\rho_{\mathrm{op}}\right)$ were estimated using BlochGruneisen (BG) model of resistivity. It is observed that the resistivity contribution due to electron-electron interaction shows typical quadratic temperature dependence. Spin fluctuation-induced resistivity is dominant over electron-electron and electron-phonon contributions in overall temperature range in the manganite nanoparticles. Resistivity in the semiconducting phase is discussed with small polaron conduction (SPC) model. SPC model consistently retraces the low-temperature resistivity behaviour $(T<53 \mathrm{~K})$. Finally, the theoretically calculated resistivity compared with experimental data is found to be consistent in wide range of temperature.
\end{abstract}

Keywords. Manganite nanoparticles; resistivity; spin fluctuation; electron-phonon interaction; electronelectron interaction; small polaron conduction.

\section{Introduction}

Nanocrystalline manganite materials are currently a focus of intense investigations because of their temperatureand size-dependent magnetoresistive effects and use in technological applications. The basic magnetic and electric transport properties of manganite materials strongly depend on temperature and size at nanometer scale and differ significantly from the properties of the bulk material (Brown 1963; Sun and Li 2001; Dutta et al 2003; Lopez-Quintela et al 2003; Jönsson 2004; Dey and Nath 2006; Petracic et al 2006). Manganite nanostructures show a sharp transition between semiconducting phase and metallic phase with change in temperature (Dutta et al 2003; Lopez-Quintela et al 2003; Dey and Nath 2006). The spin-polarized transport of conduction electrons at the grain boundaries seems to be the main reason for electro-transport properties. Manganites crystallize in perovskite structures in which $\mathrm{Mn}-\mathrm{O}-\mathrm{Mn}$ bonds

*Author for correspondence (kkchoudharyl@yahoo.com) constitute the basis of the electrical and magnetic properties.

Dutta et al (2003) have reported the effect of particle size on the magnetic and transport properties of $\mathrm{La}_{0.875}$ $\mathrm{Sr}_{0.125} \mathrm{MnO}_{3}$ nanoparticles of particle sizes 18, 36 and $50 \mathrm{~nm}$. The electrical transport properties show a strong temperature and nanoparticle size dependence, though the magnetization data of the nanoparticle samples show no sign of structural transitions. Resistivity of $\mathrm{La}_{0 \cdot 875} \mathrm{Sr}_{0 \cdot 125}$ $\mathrm{MnO}_{3}$ manganite nanoparticles decreases with temperature and shows semiconducting phase at low temperatures below $50 \mathrm{~K}$, becomes minimum at around $50-53 \mathrm{~K}$ and increases with temperature in metallic phase at higher temperatures between 53 and $170 \mathrm{~K}$. The effects of temperature and particle size on the magnetic and transport properties have been attributed to the domain status, changes in Mn-O-Mn bond angle and Mn-O bond length in comparison with bulk samples (Dutta et al 2003). The reduction in particle size has a direct consequence on the electronic and magnetic properties of a system. It is observed that as we reduce the particle size of magnetic systems, there is an increase in resistivity, showing 
insulating properties at very low particle size, and a higher value of magnetoresistance compared to the bulk samples, which is attributed to a spin-polarized intergrain tunnelling mechanism (Sun and Li 2001).

Dey and Nath (2006) have investigated the effect of nanometric grain size on magneto- and electronic-transport properties of single-phase, nanocrystalline, granular $\mathrm{La}_{0.7} \mathrm{Ca}_{0.3} \mathrm{MnO}_{3}$ having an average grain size in the nanometric regime $14-27 \mathrm{~nm}$. They observed that the resistivity increases with decrease in particle size; maximum resistivity is observed for grain size of $14 \mathrm{~nm}$, whereas it is minimum for size $27 \mathrm{~nm}$. The transition from metallic phase to semiconducting phase is observed at around 130 and $160 \mathrm{~K}$ for 17 and $21 \mathrm{~nm} \mathrm{La}{ }_{0 \cdot 7} \mathrm{Ca}_{0 \cdot 3} \mathrm{MnO}_{3}$ nanoparticles, respectively. The gradual drop of metalsemiconductor transition temperature with a decrease in grain size is also observed. Effect of temperature and grain size on electro-transport properties has been analysed using a phenomenological model based on the spin-polarized transport of conduction electrons at the grain boundaries (Dey and Nath 2006).

The single crystals and bulk polycrystalline samples of $\mathrm{La}_{1-x} \mathrm{Sr}_{x} \mathrm{MnO}_{3}$ system have been extensively studied in the low-doped regime because of the series of structural, magnetic, and electronic phase transitions they undergo as a function of temperature, which is a paradigmatic example of the interplay between carrier, lattice, spin and orbital degrees of freedom (Marques et al 2001; Martinez et al 2001; Nogues et al 2001). A fully reproducible magnetoresistance (up to 20\%) was discovered in small magnetic fields, in $\mathrm{Al}_{2} \mathrm{O}_{3} / \mathrm{CoFe} / \mathrm{Co}$ junctions (Moodera et al 1995). All these data show that new tools are now available to obtain artificial MR from metallic compounds just by manipulating the micro/nanostructure of these materials. The measured ferromagnetic-insulatorferromagnetic tunnelling was found consistent with Julliere's model based on the spin polarization of the conduction electrons of the magnetic films (Moodera et al 1995).

From the earlier results, it is concluded that finite-size effects play a crucial role in physics, nanoscale magnetism provides a wealth of scientific knowledge and potential for applications. When the size of the magnetic particles is reduced to a few nanometers, the outstanding magnetic and electric particles are exhibited as compared to their bulk counterparts. The transition of temperature-dependent electrical resistivity from semiconducting phase to metallic phase at a particular temperature is an important feature of manganite nanostructures, which needs to be addressed in two different phases. In this paper, we have presented a theoretical model to investigate the temperature dependence of resistivity of $18 \mathrm{~nm} \mathrm{La} \mathrm{La}_{0.85} \mathrm{Sr}_{0.125} \mathrm{MnO}_{3}$ manganite nanoparticles. Resistivity in metallic phase is investigated by considering the strong spin fluctuation effect along with electron-phonon and electron-electron contributions, whereas the resistivity in semiconducting phase is addressed using small polaron conduction (SPC) model. The detailed theoretical model is presented in the following section.

\section{Model}

2.1 Spin fluctuation mechanism of resistivity in metallic phase of manganite nanopaticles

It is quite reasonable to consider the long-range spin fluctuations-induced resistivity with the characteristic energy $\hbar \omega_{\mathrm{sf}}$, corresponding to an effective temperature $T_{\mathrm{sf}}=$ $\hbar \omega_{\mathrm{s}} / K_{\mathrm{B}}$. Considering the fluctuation-induced thermal broadening effects, we use a Drude-Lorentz type function for spin fluctuation contribution towards electrical resistivity (Sergeenkov et al 2007):

$$
\rho_{\mathrm{sf}}(T)=\rho_{\mathrm{res}} \int_{-\omega_{\mathrm{sf}}}^{\left(\Omega(T)-\omega_{\mathrm{sf}}\right)} \frac{\omega_{\mathrm{sf}} \mathrm{d} \omega}{\pi\left(\omega^{2}+\omega_{\mathrm{sf}}^{2}\right)},
$$

or

$$
\rho_{\text {sf }}(T)=\rho_{\text {res }}\left[\frac{1}{4}+\frac{1}{\pi} \tan ^{-1}\left(\frac{T-T_{\text {sf }}}{T_{\text {sf }}}\right)\right],
$$

where $\rho_{\text {res }}$ is the residual contribution and given by

$$
\rho_{\text {res }}=\frac{1}{\omega_{\mathrm{p}}^{2} \varepsilon_{0} \tau_{0}},
$$

here $\omega_{\mathrm{p}}$ is the plasmon frequency, $1 / \tau_{0}$ the corresponding scattering rate and $\varepsilon_{0}=8.85 \times 10^{-12} \mathrm{~F} / \mathrm{m}$ the vacuum permittivity.

The temperature dependence in (1) comes from the cut of frequency $\Omega(T)=U(T) / \hbar$, which account for spin fluctuations, with an average thermal energy $U(T)=1 / 2 C$ $\left\langle u^{2}\right\rangle=K_{\mathrm{B}} T$, where $C$ is the force constant of a twodimensional harmonic oscillator and $\left\langle u^{2}\right\rangle$ is the rms displacement of the magnetic atoms from their equilibrium positions.

We used the following expression to estimate total resistivity

$$
\rho(T)=\rho_{\mathrm{sf}}(T)+\rho_{\mathrm{e}-\mathrm{ph}}(T)+\rho_{\mathrm{e}-\mathrm{e}}(T)
$$

where the last two terms of the right-hand side of the equation are related to the electron-phonon and electronelectron contributions.

\subsection{Electron-phonon contribution to resistivity}

We anticipate that both acoustic and optical phonons participate in the process of electrical conduction. Acoustic 
phonon contribution yields the Bloch-Gruneisen function of temperature-dependent resistivity (Choudhary 2012)

$$
\begin{aligned}
\rho_{\text {a.c. }}\left(T, \theta_{\mathrm{D}}\right)= & 4 A_{\mathrm{ac}}\left(T / \theta_{\mathrm{D}}\right)^{4} \\
& \times T \int_{0}^{\theta_{\mathrm{D}} / T} x^{5}\left(e^{x}-1\right)^{-1}\left(1-e^{-x}\right)^{-1} \mathrm{~d} x .
\end{aligned}
$$

where $x=\hbar \omega / k_{\mathrm{B}} T$ and $\theta_{\mathrm{D}}$ the Debye temperature. $A_{\mathrm{ac}}$ being a constant of proportionality. The phonon spectrum can be conveniently separated into two parts of phonon density of states. Therefore, it is natural to choose a model phonon spectrum consisting of two parts: an acoustic Debye branch characterized by the Debye temperature $\theta_{\mathrm{D}}$ and an optical mode of vibration defined by the Einstein temperature $\theta_{\mathrm{E}}$. In case of the Einstein type of phonon spectrum (an optical mode) $\rho_{\text {op }}(T)$ may be described as follows

$$
\rho_{\mathrm{op}}\left(T, \theta_{\mathrm{E}}\right)=A_{\mathrm{op}} \theta_{\mathrm{E}}^{2} T^{-1}\left(e^{x}-1\right)^{-1}\left(1-e^{-x}\right)^{-1}
$$

Finally, the phonon resistivity can be conveniently modelled as

$$
\rho_{\mathrm{e}-\mathrm{ph}}(T)=\rho_{\text {a.c. }}\left(T, \theta_{\mathrm{D}}\right)+\rho_{\mathrm{op}}\left(T, \theta_{\mathrm{E}}\right),
$$

$A_{\mathrm{ac}}$ and $A_{\mathrm{op}}$ are the factors representing the strength of scattering of electrons with acoustic and optical phonons, respectively.

\subsection{Electron-electron contribution to resistivity}

The electron-electron contribution due to electronelectron scattering follows $\rho_{\mathrm{e}-\mathrm{e}}(T)=B T^{2}$ with

$$
B=\frac{K_{\mathrm{B}}^{2}}{\hbar \omega_{\mathrm{p}}^{2} \varepsilon_{0} E_{\mathrm{f}}},
$$

here $\lambda$ is the electron-phonon coupling constant and $E_{\mathrm{f}}$ the Fermi energy.

\subsection{Small polaron conduction (SPC) model of resistivity for semiconducting phase of manganite nanopaticles}

We shall now switch to a brief description of the temperature-dependent resistivity, due to adiabatic SPC model (Choudhary 2012). We must mention that the most rapid motion of a small polaron occurs when the carrier hops each time, the configuration of vibrating atoms in an adjacent site coincides with that in the occupied site. Henceforth, the charge carrier motion within the adiabatic regime is faster than the lattice vibrations and the resistivity for SPC as follows

$$
\rho=\rho_{\mathrm{os}} T \exp \left(\frac{E_{\mathrm{p}}}{k_{\mathrm{B}} T}\right),
$$

$E_{\mathrm{p}}$ is the polaron formation energy and $k_{\mathrm{B}}$ the Boltzmann constant. The resistivity coefficient $\rho_{\mathrm{os}}$ is given by

$$
\rho_{\mathrm{os}}=\frac{k_{\mathrm{B}}}{n e^{2} D},
$$

where $n$ is the charge carrier density, $e$ the electronic charge and $D$ the polaron diffusion constant.

\section{Results and discussion}

We use a Drude-Lorentz type function to evaluate spin fluctuation contribution towards electrical resistivity, along with electron-electron and electron-phonon interaction contributions in metallic phase of manganite nanoparticles. For numerical calculations of temperature-dependent resistivity of $\mathrm{La}_{0.875} \mathrm{Sr}_{0.125} \mathrm{MnO}_{3}$ manganite nanoparticles of particle size $18 \mathrm{~nm}$, we use the value of some physical parameters as $A_{\mathrm{ac}}=0.41 \times 10^{-2} \Omega \mathrm{cm} / \mathrm{K}, A_{\mathrm{op}}=1.1 \times 10^{-2}$ $\Omega \mathrm{cm} / \mathrm{K}, B=3.26 \times 10^{-6} \Omega \mathrm{cm} / \mathrm{K}^{2}$ and $\rho_{\text {res }}=0.52 \Omega \mathrm{cm}$. Here coefficients $A_{\mathrm{ac}}, A_{\mathrm{op}}$ and $B$ represent the strength of electron-acoustic phonon, electron-optical phonon and electron-electron scattering processes, respectively, whereas $\rho_{\text {res }}$ represent the strength of spin fluctuation effect on resistivity. The numerical values of mentioned parameters are optimized to fit the experimental data. The best fit method for obtaining the value of $A_{\mathrm{ac}}, A_{\mathrm{op}}$ and $B$ is consistent with the metallic conduction in nanoparticles and other materials (Thompson 1975; Varshney et al 2002; Choudhary 2012).

The relative contribution to resistivity due to electronelectron and electron-phonon interactions along with the spin fluctuation-induced resistivity of $18 \mathrm{~nm}$ nanoparticles of $\mathrm{La}_{0.875} \mathrm{Sr}_{0.125} \mathrm{MnO}_{3}$ manganite is plotted in figure 1. Features in the graph represent that the electronelectron contribution to resistivity shows typical $T^{2}$ characteristic (dashed line in figure 1) and becomes important only at higher temperatures though the relative magnitude of electron-electron contribution is very small (about 3\% of total resistivity) in comparison to electron-phonon and spin fluctuation contribution. The quadratic temperature dependence of resistivity over a wide temperature range is a cause of three-dimensional electron-electron (umklapp) scattering, its magnitude depends on the plasma frequency. This feature is consistent with many metallic conductors in which free electron gas exhibits quadratic temperature dependence of resistivity (Thompson 1975; Varshney et al 2002, 2009; Choudhary 2012). The contributions of acoustic $\left(\rho_{\mathrm{ac}}\right)$ and optical phonons $\left(\rho_{\mathrm{op}}\right)$ towards resistivity are shown separately in figure 1 . It is inferred from the curve that $\rho_{\text {ac }}$ increases linearly (dashdot line), while to that the non-linearity (exponential temperature dependence) appears from the optical phonon contribution to resistivity $\rho_{\text {op }}$ (dash-dot-dot line) because optical phonons are activated more at higher temperatures. These results are consistent with the scattering of 
electrons with acoustic and optical phonons in metallic state of various materials (Varshney et al 2002; Choudhary 2012).

Moreover, electron-electron and electron-phonon contributions to resistivity, a major source of resistivity, come from spin fluctuation mechanism in manganite nanoparticles which is responsible for colossal magneto resistance and distinguish these materials from normal metallic conductors. Spin fluctuation-induced electrical resistivity is an intrinsic property of manganite materials, which is caused by finite magnetic momentum of magnetic ions present in the materials. As compared to electron-phonon and electron-electron contributions, spin fluctuation contribution is dominant in overall temperature range; it is an artefact of the spin alignment in manganite nanoparticles (dotted line in figure 1). All three contributions (electron-electron, electron-phonon and spin fluctuation) are added to obtain total theoretically calculated resistivity (solid line in figure 1).

The electric transport properties of manganite nanoparticles are very sensitive to the particle size of nanostructures (Brown 1963; Sun and Li 2001; Dutta et al 2003; Lopez-Quintela et al 2003; Jönsson 2004; Dey and Nath 2006; Petracic et al 2006). Electrical resistivity increased with decrease in particle size in overall temperature range. The spin-polarized transport of conduction electrons at the grain boundaries seems to be the main reason for size-dependent electro-transport properties (Sun and $\mathrm{Li}$ 2001). This effect of spin-polarized tunnelling between neighbouring grains is absent in single crystals and rapidly increase in nanomaterials due to increase in interfaces (Milner et al 1996). The electric transport properties of these perovskite manganite nanoparticles are very

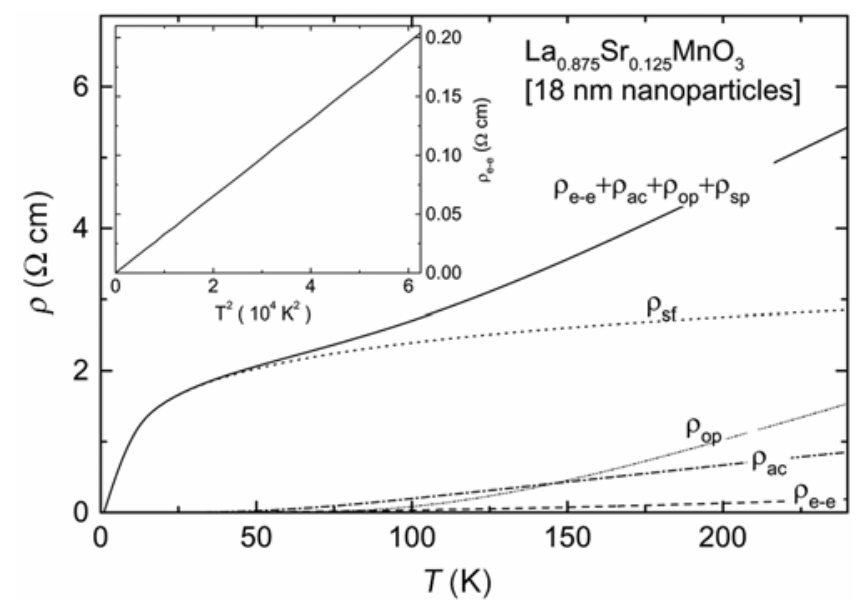

Figure 1. Resistivity contribution due to spin fluctuation effect $\rho_{\text {sf }}$ (dot), acoustic-phonon contribution $\rho_{\text {ac }}$ (dash-dot), optical-phonon contribution $\rho_{\mathrm{op}}$ (dash-dot-dot) and electronelectron interaction $\rho_{\mathrm{e}-\mathrm{e}}$ (dash) of $18 \mathrm{~nm} \mathrm{La}_{0.875} \mathrm{Sr}_{0.125} \mathrm{MnO}_{3}$ manganite nanoparticles is plotted with temperature. Inset shows electron-electron contribution to resistivity $\rho_{\mathrm{e}-\mathrm{e}}$ with square of temperature. sensitive to $\mathrm{Mn}-\mathrm{O}-\mathrm{Mn}$ bond angle and $\mathrm{Mn}-\mathrm{O}$ bond length, which also depend on the particle size. The decrease of the bond angle and the increase of the bond length affect the overlap integral between the two Mn ions and the conductivity between two grains is affected by the grain boundaries (Dutta et al 2003). The doubleexchange mechanism shows that the transfer integral for electrical conduction between neighbouring $\mathrm{Mn}$ sites is given by $t=t_{0} \cos (\theta / 2)$ where $\theta$ is the angle between the neighbouring $\mathrm{Mn}$ spins. Decrease in magnetization and increase in resistivity is observed as we decrease the particle size of perovskite nanomanganites (Balcells et al 1998; Rivas et al 2000; Yi et al 2000; Zhu et al 2001). This effect is observed because of broken Mn-O-Mn bonds at the surface of the smaller nanoparticles that hamper the exchange interaction and degrade connectivity for electron conduction.

The size dependence in the present model comes from the characteristic parameters $A_{\mathrm{ac}}, A_{\mathrm{op}}, B$ and $\rho_{\text {res }}$. These are the free parameters for the material and depend on the size and intrinsic properties of the nanoparticles. The size dependence on the phonon contribution to the resistivity of a nanostructure comes from the value of $x$ in (4), where $x=\hbar \omega / k_{\mathrm{B}} T$. Here $\omega=c / \lambda, c$ is the sound velocity averaged over all the acoustic modes and $\lambda$ is the phonon wavelength. At a given temperature $T$, the size dependence to the integral in (4) comes from the value of $x$ for which the integrand has a maximum value. The dominant value of $x$ depends on the size that decides the dominant value of $\omega$ and $\lambda$ in the test material. Here, it is instructive to mention that Bid et al (2006) have also reported the size dependence of electron-phonon resistivity by value of $\omega$ and $\lambda$ in nanostructures. Similarly, the size dependence of $\rho_{\mathrm{e}-\mathrm{e}}(T)$ is introduced with the value of electron-electron scattering parameter $B$, whose optimum value depends on dominant value of $\omega_{\mathrm{p}}$ and Fermi energy $E_{\mathrm{F}}$ in the particular nanoparticles. Further, the spin fluctuation contribution to resistivity $\rho_{\mathrm{sf}}(T)$ is proportional to $\rho_{\text {res }}$, which is a size-dependent free parameter for nanoparticles. As the size of nanoparticle decreased, the spins are confined to a smaller grains and spin polarization effect increased, which is responsible for increase in the spin fluctuation-induced resistivity and thus a higher value of $\rho_{\text {res }}$ is obtained.

Let us discuss the resistivity behaviour in low-temperature semiconducting regime $(20 \mathrm{~K}<T<53 \mathrm{~K})$ of $18-\mathrm{nm}$ $\mathrm{La}_{0.875} \mathrm{Sr}_{0.125} \mathrm{MnO}_{3}$ manganite nanoparticles. We have used the adiabatic SPC model. Keeping in mind that the charge carrier motion is faster than the atomic vibrations in the adiabatic regime and, hence, the nearest-neighbour hopping of a small polaron leads to mobility with a thermally activated form. We obtain the optimum value of polaron formation energy $E_{\mathrm{p}}=12 \mathrm{meV}$ and the diffusion coefficient as $D=3.717 \times 10^{-3} \mathrm{~cm}^{2} \mathrm{~s}^{-1}$. The value of polaron diffusion constant $D$ represents the feasibility of small polaron conduction in the test materials whose 
value is obtained by fitting method. The resistivity $\rho_{\text {SPC }}$ obtained from SPC model along with experimental data is plotted with temperature $T$ in figure 2. SPC model with realistic physical parameters successfully retraces the reported experimental behaviour of resistivity in semiconducting regime $(20 \mathrm{~K}<T<53 \mathrm{~K})$ of manganite nanostructures. The results obtained from the SPC model for resistivity are consistent with the previous reporting for nanostructures (Choudhary 2012).

Finally, the total resistivity (shown by solid line) in a wide temperature range is plotted in figure 3 along with

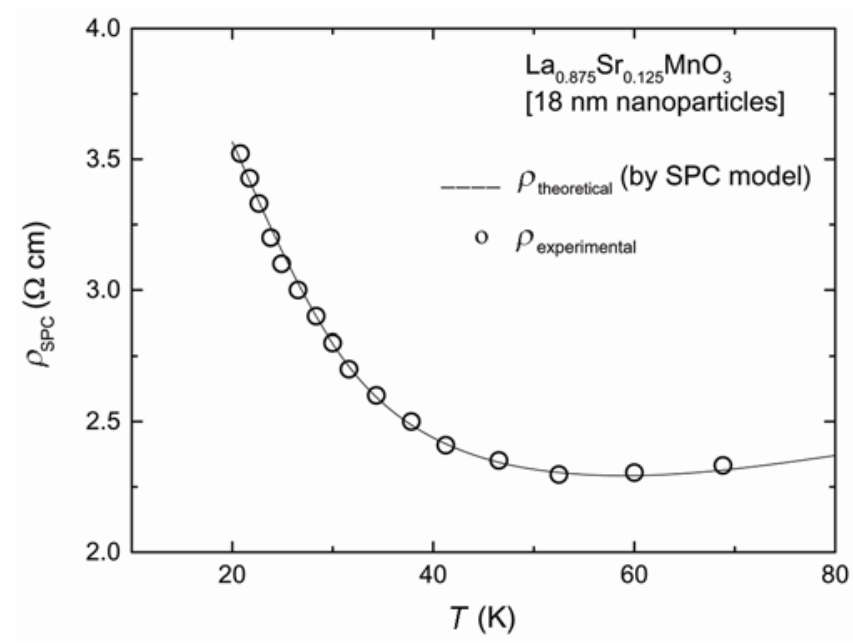

Figure 2. Variation of resistivity $\rho_{\mathrm{SPC}}(T)$ with temperature in semiconducting phase of $18-\mathrm{nm} \mathrm{La}_{0.875} \mathrm{Sr}_{0.125} \mathrm{MnO}_{3}$ manganite nanoparticles, fitted using SPC model (solid line), compared with experimental data (open circles) taken from Dutta et al (2003).

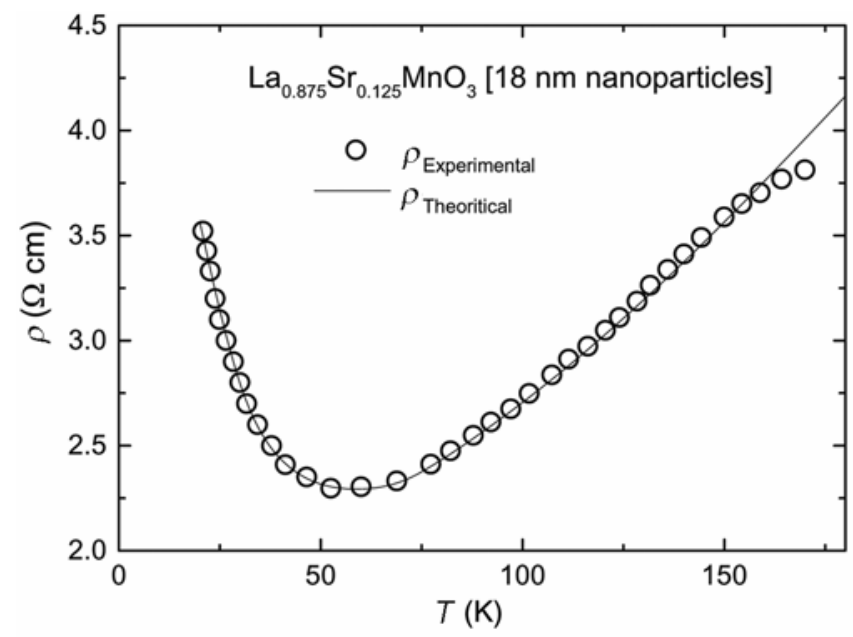

Figure 3. Variation of resistivity $\rho(T)$ of $18-\mathrm{nm} \mathrm{La}_{0.875}$ $\mathrm{Sr}_{0.125} \mathrm{MnO}_{3}$ manganite nanoparticles with temperature. Open circles are the experimental data taken from Dutta et al (2003). Solid line is the fitting by SPC model and spin fluctuation mechanism supplemented by electron-phonon and electronelectron scattering effects. the experimental data (Dutta et al 2003) (presented by open circles). Here, it is seen that the present model based on spin fluctuation mechanism of resistivity along with electron-phonon and electron-electron contributions seems to be sufficient to explain the temperature and size dependence electrical resistivity in metallic phase of nanocrystalline manganite materials, while, SPC model based on small polaron formation is successful in explaining the resistivity in semiconducting phase. Overall, the present approach successfully explains the anomalous temperature dependence resistivity in wide temperature range in both semiconducting and metallic phase of $\mathrm{La}_{0.875} \mathrm{Sr}_{0.125} \mathrm{MnO}_{3}$ manganite nanoparticles. Resistivity calculated from the present model shows good agreement with experimental data (Dutta et al 2003) in a wide temperature range. Present theoretical analysis is expected to provide guidelines to design and prepare new nano-manganite materials with desired physical properties for technological applications.

\section{Conclusions}

$\mathrm{La}_{0.875} \mathrm{Sr}_{0.125} \mathrm{MnO}_{3}$ manganite nanoparticles of $18-\mathrm{nm}$ particle size shows anomalous temperature-dependent electrical resistivity $\rho(T)$, it shows semiconducting phase in low temperature regime $(20 \mathrm{~K}<T<53 \mathrm{~K})$ and shows metallic phase at high temperatures $(53 \mathrm{~K}<T<170 \mathrm{~K})$. The resistivity in metallic phase of manganite nanoparticles has been successfully explained considering the strong spin fluctuation effect, which is modelled using Drude-Lorentz type function along with the electronphonon and electron-electron contributions. The present analysis reveals that the spin fluctuation effect is the main source of resistivity in overall temperature range. The electron-electron contribution to resistivity shows typical $T^{2}$ characteristic, which is a cause of threedimensional electron-electron (umklapp) scattering, though, the contribution from electron-electron scattering is very small as compared to electron-phonon contribution. The electron-phonon contribution is divided in linear acoustic-phonon and exponential optical-phonon contribution.

The realistic values of some characteristic parameters $A_{\mathrm{ac}}, A_{\mathrm{op}}, B$ and $\rho_{\text {res }}$ that represent the strength of electronelectron, electron-acoustic phonon, electron-optical phonon and spin fluctuation effect on resistivity are used to evaluate the model equations, these are the free parameters for the material and depend on the size and intrinsic properties of the nanoparticles. The spin-polarized transport of conduction electrons at the grain boundaries seems to be the main reason for size-dependent electrotransport properties. Spin fluctuation-induced electrical resistivity is an intrinsic property of manganite materials appear due to finite magnetic momentum of magnetic ions present in the materials. The present model 
incorporating electron-electron, electron-phonon and spin fluctuation contributions can successfully reveal the experimental data on temperature- and size-dependent electrical resistivity in metallic phase $(T>53 \mathrm{~K})$ of nanocrystalline manganite materials.

Further, we have theoretically analysed the electrical resistivity behaviour in semiconducting phase of $\mathrm{La}_{0.875}$ $\mathrm{Sr}_{0.125} \mathrm{MnO}_{3}$ manganite nanoparticles at temperatures below $53 \mathrm{~K}$ using SPC mechanism. The developed approach consistently explains the reported behaviour of temperature-dependent resistivity in wide temperature range in both metallic and semiconducting phases of manganite nanoparticles.

\section{Acknowledgement}

Financial assistance from Madhya Pradesh Council of Science and Technology, Bhopal, India, is gratefully acknowledged.

\section{References}

Balcells L, Fontcuberta J, Martínez B and Obradors X 1998 Phys. Rev. B58 R14697

Bid A, Bora A and Raychaudhary A K 2006 Phys. Rev. B74 035426

Brown W F 1963 J. Phys. Rev. 1301677

Choudhary K K 2012 J. Phys. Chem. Solids 73460

Dey P and Nath T K 2006 Phys. Rev. B73 214425
Dutta Anulekha, Gayathri N and Ranganathan R 2003 Phys. Rev. B68 054432

Jönsson P E 2004 Adv. Chem. Phys. 128191

Lopez-Quintela M A, Hueso L E, Rivas J and Rivadulla F 2003 Nanotechnology 14212

Marques R F C, Jafelicci Jr M, Paiva-Santos C O, Varanda L C and Godoi R H M 2001 J. Magn. Magn. Mater. 226-230 812

Martinez B, Laukhin V, Fontcuberta J, Nogues J, Skumryev V, Munoz J S, Pisard L and Revcolevschi A 2001 J. Appl. Phys. 896633

Milner A, Gerber A, Groisman B, Karpovsky M and Gladkikh A 1996 Phys. Rev. Lett. 76475

Moodera J S, Kinder L S, Wong T M and Meservey R 1995 Phys. Rev. Lett. 743273

Nogues J, Skumryev Vassil, Munoz J S, Martinez B, Fontcuberta J, Pinsard L and Revcolevschi A 2001 Phys. Rev. B64 024434

Petracic O, Chen X, Bedanta S, Kleemann W, Sahoo S, Cardoso S and Freitas P P 2006 J. Magn. Magn. Mater. 300192

Rivas J, Hueso L E, Fondado A, Rivadulla F and LopezQuintela M A 2000 J. Magn. Magn. Mater. 22157

Sergeenkov S, Lanfredi A J C and Araujo-Moreira F M 2007 JEPT Lett. 85592

Sun H and Li Z Y 2001 Phys. Rev. B64 224413

Thompson A H 1975 Phys. Rev. Lett. 351786

Varshney Dinesh, Choudhary K K and Singh R K 2002 Supercond. Sci. Technol. 151119

Varshney Dinesh, Shaikh M W and Mansuri I 2009 J. Alloys Compd. 486726

Yi Tao et al 2000 J. Phys. Chem. Solids 611407

Zhu T, Shen B G, Sun J R, Zhao H W and Zhan W S 2001 Appl. Phys. Lett. 783863 